\title{
GENERALIZATION OF A PROBABILITY LIMIT THEOREM OF CRAMÉR
}

BY

W. FELLER

1. Introduction. Let $\left\{X_{k}\right\}, k=1,2, \cdots$, be mutually independent random variables, that is to say independent real functions in a space in which a probability measure is defined. Let $V_{k}(x)=\operatorname{Prob}\left\{X_{k} \leqq x\right\}$ be the distribution function of $X_{k}$ and suppose that the second moment

$$
\sigma_{k}^{2}=\int_{-\infty}^{+\infty} x^{2} d V_{k}(x)
$$

exists; without loss of generality we may then assume that

$$
\int_{-\infty}^{+\infty} x d V_{k}(x)=0
$$

Let

$$
S_{n}=X_{1}+\cdots+X_{n}
$$

and

$$
s_{n}^{2}=\sigma_{1}^{2}+\cdots+\sigma_{n}^{2}
$$

Finally, let $F_{n}(x)=$ Prob $\left\{S_{n} \leqq x\right\}$ be the distribution function of $S_{n}$. The independence of the $X_{n}$ implies that

$$
F_{1}(x)=V_{1}(x), \quad F_{n+1}(x)=\int_{-\infty}^{+\infty} F_{n}(x-y) d V_{n+1}(y)
$$

The central limit theorem asserts that, under very general conditions,

$$
F_{n}\left(x s_{n}\right) \rightarrow \Phi(x)=(2 \pi)^{-1 / 2} \int_{-\infty}^{x} e^{-(1 / 2) y^{2}} d y,
$$

uniformly in every finite interval. If, however, with $n \rightarrow \infty$ also $x \rightarrow \infty$ it follows only that $F_{n}\left(x s_{n}\right) \rightarrow 1$, but it is not even clear whether $1-F\left(x s_{n}\right)$ is of the same order of magnitude as $1-\Phi(x)$. In the Bernoulli case with

$$
V_{\dot{k}}(x) \equiv V(x)=\left\{\begin{array}{llr}
0 & \text { for } & x<-p<0, \\
1-p & \text { for } & -p \leqq x<1-p, \\
p & \text { for } & 1-p \leqq x,
\end{array}\right.
$$

Presented to the Society, February 27, 1943; received by the editors March 15, 1943. 
the behavior of $F_{n}\left(x s_{n}\right)$ when $x=x(n) \rightarrow \infty$ has been the object of detailed investigations by A. Khintchine [5] $\left(^{1}\right)$, N. Smirnoff [7], P. Lévy [6] and M. Fréchet [4]. A more general case has recently been treated by $\mathrm{H}$. Cramér [2]; he considers the case of "equal components" where all $V_{k}(x)$ are identical, $V_{k}(x) \equiv V(x)$, and it is moreover supposed that the integral

$$
\int_{-\infty}^{+\infty} e^{a x} d V(x)
$$

exists for all $|a|<a_{0} \neq 0$. Nothing seems to be known in the case of unequal components. The problem as such has been stated by P. Lévy [6, p. 289].

The purpose of the present paper is to generalize Cramér's result to the case of unequal components. This generalization seems of interest in itself, but it is also to serve as the basis for the establishment of the general form of the so-called law of the iterated logarithm.

We shall consider only the case where $\left|X_{n}\right|$ has an upper bound of the form $o\left(s_{n}\right)$. Since $s_{n} \rightarrow \infty$, this restriction is not very strong. In some cases it is possible to free oneself from this restriction, provided the "tails" $\int_{|x|>\epsilon s_{n}} d V_{n}(x)$ are sufficiently small. However, in many applications the principle of equivalent sequences of random variables will automatically reduce the consideration to the case of bounded variables. Our proof is based on a straightforward adaptation of Cramér's proof, although we shall avoid the use of characteristic functions. Cramér's proof, in turn, rests on a transformation first used by F. Esscher [3] in connection with an insurance problem. In the special case of equal components our condition $\left|X_{n}\right|=o\left(s_{n}\right)$ is more restrictive than Cramér's condition that the integral (1.7) should exist. On the other hand, our results will be slightly sharper than Cramér's, since it will be possible for us to free ourselves of the logarithmic term in the remainder without introducing a new hypothesis.

2. Results. Throughout what follows we shall suppose that the $\left\{X_{k}\right\}$ are subject to the conditions (1.1) and (1.2). Moreover, we shall suppose that (cf. (1.4))

$$
\left|X_{k}\right|<\lambda_{n} s_{n}, \quad \text { for } k=1, \cdots, n \text {. }
$$

In most applications the $\left\{X_{k}\right\}$ will form an infinite sequence, and $\lambda_{n} \rightarrow 0$. Condition (2.1) can then be replaced by $\left|X_{n}\right|<\lambda_{n} s_{n}$, provided that the sequence $\lambda_{n}$ is non-increasing. It should be noted, however, that Theorem 1 is strictly a theorem on a finite sequence of random variables.

In $\S \S 3-5$ we shall prove

THEOREM 1. If

$$
0<\lambda_{n} x<\left(3-5^{1 / 2}\right) / 4
$$

(1) The numbers in brackets refer to the bibliography at the end of the paper. 
then

$$
1-F_{n}\left(x s_{n}\right)=e^{-(1 / 2) x^{2} Q_{n}(x)}\left[\{1-\Phi(x)\}+\theta \lambda_{n} e^{-(1 / 2) x^{2}}\right] .
$$

Here $\theta$ has an upper bound independent of the sequence $\left\{X_{k}\right\}$ and of $n$; furthermore

$$
Q_{n}(x)=\sum_{\nu=1}^{\infty} q_{n, \nu} x^{\nu}
$$

where the coefficient $q_{n, \nu}$ depends only on the $\nu$ first moments of $V_{1}(x), \cdots, V_{n}(x)$. If, more particularly,

$$
0<\lambda_{n} x<1 / 12
$$

then

$$
|\theta|<9
$$

and

$$
\left|q_{n, \nu}\right|<(1 / 7)\left(12 \lambda_{n}\right)^{\nu}
$$

Moreover, for any $0<i<j \leqq n$ we have

$$
\left|Q_{j}(x)-Q_{i}(x)\right| \leqq(1 / 2)\left(s_{j}^{2}-s_{i}^{2}\right) / s_{n}^{2} .
$$

Before describing the construction of $q_{n, v}$ in general we proceed to describe a few implications of Theorem 1 . First let us observe that by applying the theorem to the sequence $\left\{-X_{k}\right\}$, we obtain a corresponding theorem for negative $x$. Since however $Q_{n}(x)$ depends also on moments of odd order the behavior of $F_{n}\left(x s_{n}\right)$ for $x \rightarrow-\infty$ will, in general, be different.

Since

$$
1-\Phi(x)=(2 \pi)^{-1 / 2} x^{-1} e^{-(1 / 2) x^{2}}\left(1-\vartheta / x^{2}\right)
$$

with $0<\vartheta<1$ we can write $(2.3)$ in the form

$$
1-F_{n}\left(x s_{n}\right)=(2 \pi)^{-1 / 2} x^{-1} e^{-(1 / 2) x^{2}\left(1+Q_{n}(x)\right)}\left[1-\vartheta / x^{2}+(2 \pi)^{1 / 2} \theta \lambda_{n} x\right],
$$

which shows the order of magnitude of the remainder as compared with that of the main term.

To appreciate the meaning of the theorem consider the representative case of a uniformly bounded sequence $\left\{X_{k}\right\}$ with $s_{n}^{2} \sim n$. Then $\lambda_{n}=O\left(n^{-1 / 2}\right)$. If, then, $x=o\left(n^{1 / 6}\right)$ it is seen that $Q_{n}(x) \rightarrow 0$ and $\lambda_{n} x_{n}=o\left(n^{-1 / 3}\right)$; thus $1-F_{n}\left(x s_{n}\right)$ will be of the order of magnitude of $1-\Phi(x)$. However, if $x$ is of an order of magnitude between $n^{1 / 6}$ and $n^{1 / 4}$, the order of magnitude of $1-F_{n}\left(x s_{n}\right)$ will be that of $\{1-\Phi(x)\} e^{-(1 / 2) q_{n, 1} x^{3}}$; for an $x$ between $n^{1 / 4}$ and $n^{3 / 10}$ the term $(1 / 2) q_{n, 2} x^{4}$ will be significant also, whereas $\sum_{\nu=5}^{\infty} q_{n, \nu} x^{\nu+2} \sim 0$, and so on. Still

$$
Q_{n}(x)=o(1) \quad \text { for } x=o\left(n^{1 / 2}\right) \text {, }
$$


so that for $x=o\left(n^{1 / 2}\right)$ at least $\log \left(1-F_{n}\left(x s_{n}\right)\right) \sim \log (1-\phi(x))$. Now if $x \sim n^{1 / 2}$, the behavior is quite different. Then $\left(x^{2} / 2\right) Q_{n}(x) \sim$ const. $x^{2}$, and $\log \left(1-F\left(x s_{n}\right)\right)$ will be asymptotically equivalent to $(\alpha / 2) x^{2}$ with $\alpha \neq 1$; in other words $\log \left(1-F_{n}\left(x s_{n}\right)\right)$ will no longer be asymptotically equivalent to $\log (1-\phi(x))$. A similar change in the asymptotic behavior of $1-F_{n}\left(x s_{n}\right)$ can be observed in the general case and is of fundamental importance in connection with the law of the iterated logarithm.

For use in the same connection we note also the following simple corollary to Theorem 1 .

THEOREM 2. Let $\alpha>0$ be an arbitrary constant and let $\max (2, \alpha)<x$ $<1 /\left(100 \lambda_{n}\right)$ and $\lambda_{n}<1 / 100$. Then there exist two constants $0<a_{1}<a_{2}<1$, depending only on $\alpha$, such that

$$
a_{1}<\frac{1-F\left((x+\alpha / x) s_{n}\right)}{1-F\left(x s_{n}\right)}<a_{2} .
$$

Proof. The theorem is an easy consequence of (2.10). The term in brackets lies obviously between $1 / 2$ and $3 / 2$. From (2.7) we conclude furthermore that

$$
\begin{aligned}
\left|q_{n, \nu}(x+\alpha / x)^{\nu+2}-q_{n, \nu} x^{\nu+2}\right| & \leqq\left|q_{n, \nu}\right|(\nu+2)(x+\alpha / x)^{\nu+1} \alpha / x \\
& \leqq \frac{3 \alpha(\nu+2)}{14} \cdot\left(\frac{24}{100}^{\nu}\right),
\end{aligned}
$$

so that $\alpha \leqq\left|(x+\alpha / x)^{2}\left(1+Q_{n}(x+\alpha / x)\right)-x^{2}\left(1+Q_{n}(x)\right)\right| \leqq 3 \alpha$.

Choosing, say, $a_{2}=3 e^{-\alpha / 2}$ and $a_{1}=(1 / 5) e^{-3 \alpha / 2}$ the theorem follows.

Theorem 2 is essentially equivalent to the following more elegant theorem which has been discovered by Khintchine [5] in the case of the binomial and generalized by Cramér [2] to the case of equal components.

THEOREM 3. If $x \rightarrow \infty, x=o\left(1 / \lambda_{n}\right)$, then

$$
\lim _{n \rightarrow \infty} \frac{1-F_{n}\left((x+\alpha / x) s_{n}\right)}{1-F_{n}\left(x s_{n}\right)}=\lim _{n \rightarrow \infty} \frac{1-\Phi(x+\alpha / x)}{1-\Phi(x)}=e^{-\alpha} .
$$

The proof is similar to the preceding one.

We proceed, finally, to describe the construction of the function $Q_{n}(x)$. $\operatorname{Let}\left({ }^{2}\right)$

$$
b_{k, \nu}=\int_{-\infty}^{+\infty} x^{\nu} d V_{k}(x)
$$

and define quantities $\gamma_{k_{, \nu}}$ (Thiele's semi-invariants of $V_{k}(x)$ ) by

$$
\log \int_{-\infty}^{+\infty} e^{h y} d V_{k}(y)=\sum_{\nu=2}^{\infty} \gamma_{k, \nu} \frac{h^{\nu}}{\nu !} ;
$$

(2) Note that here as in the following the integral actually extends only over the interval $|x|<\lambda_{n} s_{n}$. 
(we have in particular

$$
\begin{gathered}
\gamma_{k, 2}=b_{k, 2}, \quad \gamma_{k, 3}=b_{k, 3}, \quad \gamma_{k, 4}=b_{k, 4}-3 b_{k, 2}^{2}, \\
\gamma_{k, 5}=b_{k, 5}-10 b_{k, 2} b_{k, 3}, \cdots
\end{gathered}
$$

while $\gamma_{k, 1}=0$ by (1.2)). We put

$$
\Gamma_{n, \nu}=\sum_{k=2}^{n} \gamma_{k, v}
$$

Then

$$
\frac{x^{2}}{2}+\frac{x^{2}}{2} Q_{n}(x)=\sum_{\nu=2}^{\infty} \Gamma_{n, \nu} \frac{\nu-1}{\nu !} h^{\nu}
$$

where $h=h(x)$ is defined as the inverse function of

$$
x=\frac{1}{s_{n}} \sum_{\nu=2}^{\infty} \Gamma_{n, \nu} \frac{h^{\nu-1}}{(\nu-1) !} .
$$

For the first two coefficients we obtain

$$
\begin{aligned}
q_{n, 1} & =\frac{1}{3 s_{n}^{3}} \sum_{k=1}^{n} b_{k, 3}, \\
q_{n, 2} & =\frac{1}{12 s_{n}^{4}} \sum_{k=1}^{n} b_{k, 4}-\frac{1}{4 s_{n}^{4}} \sum_{k=1}^{n} b_{k, 2}^{2}-\frac{1}{4 s_{n}^{6}}\left(\sum_{k=1}^{n} b_{k, 3}\right)^{2} .
\end{aligned}
$$

3. A transformation. Following Esscher and Cramér we shall introduce a transformation which will reduce $\left\{V_{k}(x)\right\}$ to a new sequence of distribution functions $\left\{\bar{V}_{k}(x)\right\}$ for which the central limit theorem will become applicable. For this purpose we introduce a real parameter $h>0$ which will later on be specialized as a function of $x$ and $n$.

We put

$$
p_{k}=\int_{-\infty}^{+\infty} e^{h x} d V_{k}(x),
$$

and

$$
\bar{V}_{k}(x)=\frac{1}{p_{k}} \int_{-\infty}^{x} e^{h y} d V_{k}(y) .
$$

Then $\bar{V}_{k}(x)$ is again a distribution function. Moreover, $\bar{V}_{k}(x)$ obviously varies only within a finite interval. Hence the moments

$$
\bar{m}_{k}=\int_{-\infty}^{+\infty} x d \bar{V}_{k}(x)
$$


and

$$
\bar{\sigma}_{k}^{2}=\int_{-\infty}^{+\infty}\left(x-\bar{m}_{k}\right)^{2} d \bar{V}_{k}(x)=\int_{-\infty}^{+\infty} x^{2} d \bar{V}_{k}(x)-\bar{m}_{k}^{2}
$$

exist. In a manner analogous to (1.4) and (1.5) we define

$$
\begin{aligned}
\bar{M}_{n} & =\bar{m}_{1}+\cdots+\bar{m}_{n}, \\
\bar{s}_{n}^{2} & =\bar{\sigma}_{1}^{2}+\cdots+\bar{\sigma}_{n}^{2},
\end{aligned}
$$

and

$$
\begin{aligned}
\bar{F}_{1}(x) & =\bar{V}_{1}(x), \\
\bar{F}_{n+1}(x) & =\int_{-\infty}^{+\infty} \bar{F}_{n}(x-y) d \bar{V}_{n+1}(y) .
\end{aligned}
$$

Now by (3.2)

$$
\int_{-\infty}^{x} e^{-h y} d \bar{V}_{k}(y)=\frac{1}{p_{k}} \int_{-\infty}^{x} d V_{k}(y)=\frac{1}{p_{k}} V_{k}(x) .
$$

Hence it is seen that

$$
F_{n}(x)=p_{1} \cdots p_{n} \int_{-\infty}^{x} e^{-h y} d \bar{F}_{n}(y)
$$

holds for $n=1$; using (1.5) and (3.7) it is seen that (3.9) holds generally. Since $F_{n}(x)$ is a distribution function we have, letting $x \rightarrow \infty$,

$$
1-F_{n}(x)=p_{1} \cdots p_{n} \int_{x}^{\infty} e^{-h y} d \bar{F}_{n}(y) .
$$

Now it is our purpose to apply the central limit theorem to $\bar{F}_{n}(x)$. It will therefore be convenient to write (3.10) in the form

$$
1-F_{n}(x)=p_{1} \cdots p_{n} \int_{\left(x-\bar{M}_{n}\right) / \delta_{n}}^{\infty} e^{-h\left(\bar{M}_{n}+y \bar{s}_{n}\right)} d \bar{F}_{n}\left(\bar{M}_{n}+y \bar{s}_{n}\right) .
$$

This formula will be used for $x=\bar{M}_{n}$ only. Introducing the quantity

$$
P_{n}=\sum_{k=1}^{n} \log p_{k}
$$

we deduce from (3.11) finally

$$
1-F_{n}\left(\bar{M}_{n}\right)=e^{P_{n}-h \bar{M}_{n}} \int_{0}^{\infty} e^{-h y \bar{\sigma}_{n}} d \bar{F}_{n}\left(\bar{M}_{n}+y \bar{\xi}_{n}\right) .
$$


4. Some estimates. Operating for the moment in a purely formal manner we have by (2.13) and (3.1)

$$
\log p_{k}=\sum_{\nu=2}^{\infty} \gamma_{k, \nu} \frac{h^{\nu}}{\nu !}
$$

A simple computation shows that (cf. (3.3) and (3.4))

$$
\begin{aligned}
& \bar{m}_{k}=\frac{d \log p_{k}}{d h}=\sum_{\nu=2}^{\infty} \gamma_{k, \nu} \frac{h^{\nu-1}}{(\nu-1) !}, \\
& \bar{\sigma}_{k}^{2}=\frac{d^{2} \log p_{k}}{d h^{2}}=\sum_{\nu=2}^{\infty} \gamma_{k, \nu} \frac{h^{\nu-2}}{(\nu-2) !} .
\end{aligned}
$$

Using (2.16), (3.5), and (3.6) we obtain

$$
\begin{aligned}
P_{n} & =\sum_{\nu=2}^{\infty} \Gamma_{n, \nu} \frac{h^{\nu}}{\nu !}, \\
\bar{M}_{n} & =\sum_{\nu=2}^{\infty} \Gamma_{n, \nu} \frac{h^{\nu-1}}{(\nu-1) !}, \\
\bar{\xi}_{n}^{2} & =\sum_{\nu=2}^{\infty} \Gamma_{n, \nu} \frac{h^{\nu-2}}{(\nu-2) !} .
\end{aligned}
$$

We now proceed to find majorating power series for these expressions. It follows from (2.1) and (2.12) that for $k=1, \cdots, n$

$$
\left|b_{k, \nu}\right| \leqq \sigma_{k}^{2}\left(\lambda_{n} s_{n}\right)^{\nu-2} \leqq\left(\lambda_{n} s_{n}\right)^{\nu}
$$

Now by (1.2)

$$
p_{k}=1+\sum_{\nu=2}^{\infty} b_{k, \nu} \frac{h^{\nu}}{\nu !}
$$

We shall actually use only values $0<\lambda_{n} s_{n} h<1 / 5$, so that $p_{k}$ remains near 1 . It follows from (4.1) and (4.7) that

$$
\begin{aligned}
\sum_{\nu=2}^{\infty} \gamma_{k, \nu} \frac{h^{\nu}}{\nu !} & \prec-\log \left\{1-\frac{\sigma_{k}^{2}}{\lambda_{n}^{2} s_{n}^{2}} \sum_{\nu=2}^{\infty} \frac{\left(\lambda_{n} s_{n} h\right)^{\nu}}{\nu !}\right\} \\
& =-\log \left\{1-\frac{\sigma_{k}^{2}}{\lambda_{n}^{2} s_{n}^{2}}\left(e^{\lambda_{n} \varepsilon_{n} h}-1-\lambda_{n} s_{n} h\right)\right\},
\end{aligned}
$$

where the sign $\prec$ indicates that the power series on the left is majorated by that on the right. Now write for the moment 


$$
-\log \left\{1-\left(e^{x}-1-x\right)\right\}=\sum_{\nu=2}^{\infty} \rho_{\nu} \frac{x^{\nu}}{\nu !}
$$

so that $\rho_{2}=1, \rho_{3}=1, \rho_{4}=4, \rho_{5}=11, \rho_{\nu} \geqq 0$. Differentiating (4.10) twice we obtain

$$
\frac{x e^{x}+1}{\left(2+x-e^{x}\right)^{2}}=\sum_{\nu=2}^{\infty} \rho_{\nu} \frac{x^{p-2}}{(\nu-2) !} .
$$

Substituting here $x=1 / 2$ we obtain easily that

whence

$$
\sum_{\rho=6}^{\infty} \rho_{\nu} \frac{1}{(\nu-2) !}(1 / 2)^{\nu-2}<3 / 10
$$

$$
0 \leqq \rho_{\nu} /(\nu-2) ! \leqq(1 / 2) \cdot 2^{\nu-2}
$$

for $\nu \geqq 3$.

Comparing (4.9) with (4.10) and noting that $\sigma_{k}^{2}<\lambda_{n}^{2} s_{n}^{2}$ we deduce readily that

$$
\left|\boldsymbol{\gamma}_{k, \nu}\right| \leqq\left(\sigma_{k}^{2} /\left(\lambda_{n} s_{n}\right)^{2}\right)_{\nu}\left(\lambda_{n} s_{n}\right)^{\nu}, \quad \quad \nu \geqq 3,
$$

or by (4.12)

$$
(1 /(\nu-2) !)\left|\gamma_{k, \nu}\right| \leqq(1 / 2) \sigma_{k}^{2}\left(2 \lambda_{n} s_{n}\right)^{\nu^{-2}}, \quad \nu \geqq 3 .
$$

Accordingly we have by $(2.15)$

$$
(1 /(\nu-2) !)\left|\Gamma_{n, \nu}\right| \leqq\left(s_{n}^{2} / 2\right)\left(2 \lambda_{n} s_{n}\right)^{\nu-2}, \quad \nu \geqq 3,
$$

whilst $\Gamma_{n, 2}=s_{n}^{2}$; this allows us to deduce majorating expressions for $P_{n}, \bar{M}_{n}$ and $\bar{s}_{n}^{2}$ (cf. (4.4)-(4.6)).

5. Application of the central limit theorem. Using (4.14) and (4.2) it follows that

$$
\left|\bar{m}_{k}\right| \leqq \sigma_{k}^{2} h\left(1+\frac{1}{2} \frac{\lambda_{n} s_{n} h}{1-2 \lambda_{n} s_{n} h}\right) \leqq \lambda_{n}^{2} s_{n}^{2} h\left(1+\frac{1}{2} \frac{\lambda_{n} s_{n} h}{1-2 \lambda_{n} s_{n} h}\right)
$$

observing that $\Gamma_{n, 2}=s_{n}^{2}$ it follows similarly from (4.6) that

$$
\left|\bar{s}_{n}^{2}-s_{n}^{2}\right| \leqq \sum_{\nu=3}^{\infty}\left|\Gamma_{n, \nu}\right| \frac{h^{\nu-2}}{(\nu-2) !} \leqq s_{n}^{2} \cdot \frac{\lambda_{n} s_{n} h}{1-2 \lambda_{n} s_{n} h} .
$$

Now, by definition, $\bar{V}_{k}(x)(k=1, \cdots, n)$ varies only within the interval $|x|<\lambda_{n} s_{n}$. Accordingly, if we restrict $\lambda_{n} s_{n} h$ to an interval $0<\lambda_{n} s_{n} h<c<1 / 2$, $\bar{V}_{k}(x)$ will not vary outside of an interval centered at $\bar{m}_{k}$ and of length $O\left(\lambda_{n} \bar{s}_{n}\right)$. It follows (A. C. Berry [1, Theorem 4]) that (cf. (1.6))

$$
\left|\bar{F}_{n}\left(\bar{M}_{n}+x \bar{s}_{n}\right)-\Phi(x)\right|=O\left(\lambda_{n}\right) .
$$

More precisely let us suppose that, say, 


$$
0<\lambda_{n} s_{n} h<1 / 10 .
$$

Then

$$
\left|\bar{s}_{n}^{2} / s_{n}^{2}-1\right|<1 / 8, \text { and }\left|\bar{m}_{k}\right|<(17 / 160) \lambda_{n} s_{n} .
$$

Therefore for $k=1, \cdots, n$

$$
\begin{aligned}
\int_{|x|>(6 / 5) \lambda_{n} \bar{s}_{n}} d \bar{V}_{k}\left(x+\bar{m}_{k}\right) & =\frac{1}{p_{k}} \int_{\left|x-\bar{m}_{k}\right|>(6 / 5) \lambda_{n} \bar{s}_{n}} e^{h x} d \bar{V}_{k}(x) \\
& \leqq \frac{1}{p_{k}} \int_{|x|>\lambda_{n} \delta_{n}} e^{h x} d V k(x)=0
\end{aligned}
$$

Accordingly, using Berry's theorem, we have

$$
\bar{F}_{n}\left(\bar{M}_{n}+x \bar{s}_{n}\right)=\Phi(x)+R_{n}(x)
$$

with

$$
\left|R_{n}(x)\right|<(13 / 3) \lambda_{n}
$$

Introducing (5.4) into (3.13) we obtain

$$
1-F_{n}\left(\bar{M}_{n}\right)=e^{P_{n}-h \bar{M}_{n}}\left\{(2 \pi)^{-1 / 2} \int_{0}^{\infty} e^{-h \bar{s}_{n} y-(1 / 2) y^{2}} d y+\int_{0}^{\infty} e^{-h \bar{s}_{n}^{\prime}} d R_{n}(y)\right\}
$$

Now, using (5.5) we obtain

$$
\left|\int_{0}^{\infty} e^{-h \bar{s}_{n} y} d R_{n}(y)\right|=\left|-R_{n}(0)+h \bar{s}_{n} \int_{0}^{\infty} e^{-h \bar{s}_{n} y} R_{n}(y) d y\right| \leqq(26 / 3) \lambda_{n}
$$

(which result can be derived also by means of the second mean value theorem). The last inequality together with (5.6) shows that

$$
1-F_{n}\left(\bar{M}_{n}\right)=e^{P_{n}-h \bar{M}_{n}}\left\{e^{(1 / 2)\left(h \bar{s}_{n}\right)^{2}}\left(1-\Phi\left(h \bar{s}_{n}\right)\right)+\theta_{1} \lambda_{n}\right\}
$$

where

$$
\left|\theta_{1}\right|<26 / 3 \text {. }
$$

We now have to determine $h$ so that

$$
\bar{M}_{n} \equiv \sum_{\nu=2}^{\infty} \Gamma_{n, \nu} \frac{h^{\nu-1}}{(\nu-1) !}=x s_{n} .
$$

We write the inverse series formally,

$$
h=\frac{1}{s_{n}} \sum_{\nu=1}^{\infty} c_{n, \nu} x^{\nu}=\frac{1}{s_{n}}\left(x-\frac{x^{2}}{2 s_{n}^{3}} \sum_{k=1}^{n} b_{k, 3}+\cdots\right) .
$$

Using Cauchy's principle for majorating series and the estimate (4.15) it is 
readily seen that the formal inversion of (5.9) is possible at least for all values of $x$ for which the inversion of

$$
x=s_{n} h-\frac{1}{4} \sum_{\nu=3}^{\infty}\left(2 \lambda_{n}\right)^{\nu-2}\left(s_{n} h\right)^{n-1}
$$

is possible. The substitution

$$
\lambda_{r} x=u, \quad \lambda_{n} s_{n} h=z
$$

reduces (5.11) to

$$
u=z-\frac{1}{8} \sum_{v=2}^{\infty}(2 z)^{\nu}=z-\frac{1}{2} \frac{z^{2}}{1-2 z} ;
$$

the inverse function

$$
z=\frac{1+2 u-\left|1-6 u+4 u^{2}\right| 1 / 2}{5}=u+\sum_{v=2}^{\infty} c_{\nu}^{*} u^{\nu}
$$

is regular in the interval (2.2). Using an inductive argument we readily deduce from the series-expansion in (5.13) that

$$
\left.\frac{d^{\nu} z}{d u^{\nu}}\right|_{u=0} \geqq 0
$$

for all $\nu$. Hence $c_{\nu}^{*} \geqq 0$; we have in particular $c_{2}^{*}=1 / 2$ and $c_{3}^{*}=3 / 2$. For $u=1 / 6$ we obtain $z=1 / 5$ and hence

$$
\sum_{\nu=4}^{\infty} c_{\nu}^{*} \sigma^{-v}=1 / 80
$$

accordingly we have for $\nu \geqq 2$

$$
0 \leqq c_{v}^{*} \leqq(1 / 12) 6^{n-1} \text {. }
$$

Returning to the original variables $x$ and $h$ it follows that for $\nu \geqq 2$

$$
\left|c_{n, \nu}\right| \leqq(1 / 12)\left(6 \lambda_{n}\right)^{n-1}
$$

and

$$
s_{n} h-x<\frac{x}{12} \frac{6 \lambda_{n} x}{1-6 \lambda_{n} x} .
$$

We note in particular that the restriction of $x$ to the interval (2.5) implies that

$$
\lambda_{n} s_{n} h \leqq 13 / 144<1 / 10
$$

so that (5.3) holds; this justifies the numerical estimate of $\$ 4$.

For $u=\left(3-5^{1 / 2}\right) / 4$ we obtain from $(5.14) z=(1 / 2)\left(1-5^{-1 / 2}\right)<1 / 2$. Hence 
to values of $x$ in the interval (2.2) there correspond values of $h<1 /\left(2 \lambda_{n} s_{n}\right)$; by (4.15) these values are within the interval of convergence of the power series

$$
h \bar{M}_{n}-P_{n}=\sum_{\nu=2}^{\infty} \Gamma_{n, \nu} \frac{\nu-1}{\nu !} h^{\nu}
$$

Hence we can write

$$
h \bar{M}_{n}-P_{n}=\frac{x^{2}}{2}+\frac{x^{2}}{2} Q_{n}(x)=\frac{x^{2}}{2}+\frac{x^{2}}{2} \sum_{\nu=1}^{\infty} q_{n, \nu} x^{\nu},
$$

where the last series converges at least within (2.2). Now define a new power series

$$
Q_{n}^{*}(x)=\sum_{\nu=2}^{\infty} q_{n, \nu}^{*} x^{\nu}
$$

by substituting

$$
s_{n}^{*} h^{*}=x+\frac{x}{12} \frac{6 \lambda_{n} x}{1-6 \lambda_{n} x}
$$

into

$$
\frac{x^{2}}{2}+\frac{x^{2}}{2} Q_{n}^{*}(x)=\frac{\left(s_{n}^{*} h^{*}\right)^{2}}{2}+\frac{s_{n}^{* 2} h^{* 2}}{6} \sum_{\nu=3}^{\infty}\left(2 \lambda_{n} s_{n}^{*} h^{*}\right)^{n-2}
$$

It has been shown that the series (5.21) and (5.22) are dominating series for $s_{n} h$ and for $h \bar{M}_{n}-P_{n}$, respectively. Hence (5.22) dominates the series (5.19) so that $\left|q_{n, \nu}\right| \leqq q_{n, \nu}^{*}$. However, an easy computation shows that $1+Q_{n}^{*}\left(1 / 12 \lambda_{n}\right)<1.26$ and that $q^{*}{ }_{1}=5 \lambda_{n} / 3$; it follows that $q_{n, \nu}^{*}<(1 / 7)\left(12 \lambda_{n}\right)^{\nu}$, which proves that $Q_{n}(x)$ has the properties stated in Theorem 1 .

Now by (5.7), (5.9) and (5.19)

(5.23) $1-F_{n}\left(x s_{n}\right)=e^{(1 / 2) x^{2}\left(1+Q_{n}(x)\right)}\left\{e^{(1 / 2)\left(h \bar{s}_{n}\right)^{2}}\left(1-\Phi\left(h \bar{s}_{n}\right)\right)+\theta_{1} \lambda_{n}\right\}$.

It remains to show that here $h \bar{s}_{n}$ can be replaced by $x=\bar{M}_{n} / s_{n}$. Comparing the power series for $h \bar{s}_{n}$ and $\bar{M}_{n} / s_{n}$ it is seen that the terms of first and second order in $h$ are the same; hence $h \xi_{n}=x+O\left(x^{3} \lambda_{n}^{2}\right)$; more precisely we have

$$
\left|h \bar{s}_{n}-x\right|<2.45 \lambda_{n}^{2} x^{3}
$$

provided only that (2.5) holds. Now let for the moment

$$
f(t)=e^{(1 / 2) t^{2}}\{1-\Phi(t)\} \text {. }
$$

Then 


$$
f^{\prime}(t)=-(2 \pi)^{-1 / 2}+t e^{(1 / 2) t^{2}}\{1-\Phi(t)\},
$$

and a well known asymptotic expansion shows that

$$
0 \leqq-f^{\prime}(t) \leqq 1 /(2 \pi)^{1 / 2} t^{2}
$$

Accordingly by (5.24)

$$
\left|f\left(h \bar{s}_{n}\right)-f(x)\right|<\frac{2.45}{(2 \pi)^{1 / 2}} \frac{\lambda_{n}^{2} x^{3}}{\left(x-2.6 \lambda_{n}^{2} x^{3}\right)^{2}} \leqq \lambda_{n}^{2} x .
$$

Thus we have by (5.23) and (5.25)

$$
1-F_{n}\left(x s_{n}\right)=e^{-(1 / 2) x^{2}\left(1+Q_{n}(x)\right)}\left\{f(x)+\theta_{1} \lambda_{n}+\vartheta \lambda_{n}\right\},
$$

where $|\vartheta|<\lambda_{n} x$ and $\left|\theta_{1}\right|<26 / 3$ (cf. (5.8)).

This proves the main part of Theorem 1 . It remains only to prove (2.8). Now it follows from (2.15) and (2.16) that

$$
x^{2}\left(Q_{j}(x)-Q_{i}(x)\right)=\sum_{\nu=2}^{\infty} \frac{h^{\nu}}{(\nu-2) ! \nu} \sum_{k=i+1}^{j} \gamma_{k, \nu}
$$

Accordingly, using (4.14),

$$
\begin{aligned}
x^{2}\left|Q_{j}(x)-Q_{i}(x)\right| & \leqq \frac{h^{2}}{4} \sum_{v=2}^{\infty} 2\left(\lambda_{n} s_{n} h\right)^{\nu-2} \sum_{k=i+1}^{j} \sigma_{k}^{2} \\
& =\frac{h^{2}}{4}\left(s_{i}^{2}-s_{i}^{2}\right) \frac{1}{1-2 \lambda_{n} s_{n} h} .
\end{aligned}
$$

This implies (2.8) since $s_{n} h / x<13 / 12$ and $\lambda_{n} s_{n} h<1 / 10$ by (5.16) and (5.17), respectively.

\section{BibliograPhy}

1. A. C. Berry, The accuracy of the Gaussian approximation to the sum of independent variates. Trans. Amer. Math. Soc. vol. 49 (1941) pp. 122-136.

2. H. Cramér, Sur un nouveau théoreme-limite de la thêorie des probabilités. Actualités Scientifiques, No. 736, Lecture at the Colloque consacré à la Theorie des Probabilités. III. Paris, 1938, pp. 5-23.

3. F. Esscher, On the probability function in the collective theory of risk. Skandinavisk Aktuarietidskrift vol. 15 (1932) pp. 175-195.

4. M. Fréchet, Recherches thêoriques modernes sur la théorie des probabilités. Livre I, Paris, 1937, chap. $2, \$ 5$.

5. A. Khintchine, Ueber einen neuen Grenzwertsatz der Wahrscheinlichkeitsrechnung. Math. Ann. vol. 101 (1929) pp. 745-752.

6. P. Lévy, Théorie de l'addition des variables aléatoires. Paris, 1937, chap. 8.

7. N. Smirnoff, Ueber Wahrscheinlichkeiten grosser Abweichungen. Recueil Société Mathématique Moscou (Matematiceski Zbornik) vol. 40 (1933) pp. 441-454.

\section{Brown UNIVERSITY,}

Providence, R. I. 